\title{
An Empiric Risk Score to Guide PrEP Targeting Among MSM in Coastal Kenya
}

\author{
Elizabeth Wahome ${ }^{1}$ (D) Alexander N. Thiong' $\mathrm{o}^{1} \cdot$ Grace Mwashigadi $^{2} \cdot$ Oscar Chirro $^{1} \cdot$ Khamisi Mohamed $^{1}$. \\ Evans Gichuru' ${ }^{1}$ John Mwambi ${ }^{1}$. Matt A. Price ${ }^{3,4}$. Susan M. Graham ${ }^{1,5}$. Eduard J. Sanders ${ }^{1,6}$
}

Published online: 16 May 2018

(c) The Author(s) 2018

\begin{abstract}
Men who have sex with men (MSM), who have heterogeneous HIV-acquisition risks are not specifically targeted in Kenyan pre-exposure prophylaxis (PrEP) guidelines. We used data from an open cohort, which followed 753 initially HIV-negative MSM participants for more than 1378.5 person-years, to develop an empiric risk score for targeting PrEP delivery. Independent predictors of incident HIV-1 infection in this cohort were an age of 18-24 years, having only male sex partners, having receptive anal intercourse, having any unprotected sex, and having group sex. Poisson model coefficients were used to assign a numeric score to each statistically significant predictor. A risk score of $\geq 1$ corresponded to an HIV-1 incidence of $\geq 2.2$ [95\% confidence interval (CI) 1.2-4.1] and identified $81.3 \%$ of the cohort participants as being at high risk for HIV-1 acquisition. The area under the receiver operating characteristic curve was 0.76 (95\% CI 0.71-0.80). This empiric risk score may help Kenyan health care providers to assess HIV-1 acquisition risk and encourage PrEP uptake by high-risk MSM.
\end{abstract}

Keywords PrEP $\cdot$ Risk score $\cdot$ MSM $\cdot$ HIV-1 acquisition $\cdot$ Kenya

\section{Introduction}

Pre-exposure prophylaxis (PrEP) is effective in reducing the risk of HIV-1 acquisition [1], and has been recommended by the World Health Organization for populations that have an HIV-1 incidence of 3 per 100 person-years (PY) or higher [2]. In 2016, the Kenyan guidelines for use of antiretroviral drugs for treating and preventing HIV infection recommended PrEP for individuals who have significant risk for acquiring HIV-1 infection [3]. Although "significant risk" in the Kenyan PrEP guidelines is defined as reporting a wide

Elizabeth Wahome

ewahome@kemri-wellcome.org

1 KEMRI/Wellcome Trust Research Programme Centre for Geographic Medicine Research-Coast, Kilifi, Kenya

2 Simon Fraser University, Vancouver, Canada

3 International AIDS Vaccine Initiative, New York, USA

4 Department of Epidemiology and Biostatistics, University of California, San Francisco, San Francisco, CA, USA

5 University of Washington, Seattle, WA, USA

6 Nuffield Department of Medicine, University of Oxford, Headington, UK range of risk behaviors, anal intercourse is not specifically mentioned. In May 2017, Kenya became the second country in Africa - the first was South Africa-to roll out PrEP nationally through the National AIDS \& STI Control Programme (NASCOP) [4, 5], thereby making PrEP available to individuals who meet eligibility criteria in the published guidelines.

The Kenya PrEP program targets individuals who report any of the following: a sexual partner who is HIV-1 positive or has unknown HIV-1 status, transactional sex, a recent sexually transmitted infection (STI), recurrent use of postexposure prophylaxis, having sex while under the influence of alcohol, inconsistent condom use, and injection drug use during which needles and syringes are shared [3]. Although many men who have sex with men (MSM) may meet these eligibility criteria, the PrEP guidelines do not specifically target known risk factors for HIV-1 acquisition among MSM, including condomless anal intercourse, group sex, and the biological sex of sexual partners.

High HIV-1 and STI risks have been documented among adult male sex workers and MSM in Kenya [6-10], with estimates of HIV-1 incidence as high as 35.2 [95\% confidence interval (CI) 23.8-52.1] per 100 PY among MSM who have sex with men only [7]. Receptive anal intercourse (RAI) 
and group sex have also been identified as independent predictors of HIV-1 acquisition in this population. The risk of acquiring HIV-1 was eightfold to tenfold higher among MSM who reported having RAI, compared with those who did not [7, 9], and was twofold higher among MSM who reported having group sex, compared with those who did not [7].

Targeting PrEP initiation among MSM at highest risk for HIV-1 acquisition would optimize any impacts on HIV-1 transmission [11, 12], if these men can be reached and supported. In a secondary analysis of the iPrEX trial, MSM and transgender women who practiced RAI without a condom in the 3 months before enrollment accounted for $64 \%$ of new infections in this high-risk cohort [13]. Several screening tools have been developed to identify those at high risk for HIV-1 acquisition, including serodiscordant couples [14, 15], young women [16], sexually active men and women [17], and pregnant and postpartum women [18] in Africa. While tools to assess HIV-acquisition risk among MSM have been developed for use in well-resourced settings [19-23], no risk scoring tool is presently available to guide PrEP uptake by MSM in sub-Saharan Africa. As current PrEP eligibility criteria in Kenya's NASCOP guidelines have not been validated among this population, we set out to develop an empiric risk score to assess HIV-1 acquisition risk among MSM in coastal Kenya. We envisaged that this risk score could help Kenyan health care providers to target PrEP initiation among high-risk MSM.

\section{Methods}

\section{Study Design, Setting, and Participants}

Since July 2005, individuals residing on the Kenyan coast who are at high risk for HIV-1 acquisition have been prospectively recruited for an open cohort study on HIV-1 vaccine trial feasibility. Participants were identified for recruitment into the study by $10-15$ trained peer mobilizers who approached individuals through personal networks and at venues where they meet to establish contact with sexual partners and clients [7]. Men aged 18-49 years were eligible if they reported having had anal sex with a man in the 3 months prior to enrollment [7].

\section{Study Procedures}

Detailed cohort procedures are described elsewhere [7]. In brief, enrollment and follow-up included a face-to-face interview using a standardized risk-behavior questionnaire, HIV-1 testing and counseling, risk-reduction counseling, medical history, and physical examination at each visit. Through 2015, follow-up was quarterly for most participants and monthly for men who reported having RAI. Beginning in 2016, all participants had monthly follow-up visits. Treatment for genital symptoms suggestive of STIs and minor illnesses was provided free of charge, and hepatitis B vaccination was provided from 2009 onward [24]. Patients who had genital symptoms were treated syndromically, and laboratory-diagnosed infections were treated according to Kenya Ministry of Health recommendations. Since 2016, participants who had a gonorrhea infection were given directly observed treatment with oral cefixime $(400 \mathrm{mg})$ and azithromycin $(2 \mathrm{mg})$. Free condoms and water-based lubricants were also provided at each visit.

\section{Laboratory Methods}

HIV-1 testing was performed at each study visit using two rapid antibody test kits (Determine, Abbott Laboratories; Unigold, Trinity Biotech) in parallel. Discordant rapid HIV-1 test results were resolved using an enzyme-linked immunosorbent assay (ELISA) (Genetic System HIV-1/2 plus O EIA, Bio-Rad Laboratories). All HIV-1 negative samples were tested for p24 antigen (Vironostika HIV-1 p24 ELISA, Biomérieux) through 2015, or for HIV-1 RNA (Xpert ${ }^{\circledR}$ HIV-1 Qual, Cepheid) since 2016. Pre- and post-seroconversion samples were tested for HIV-1 RNA level (Amplicor Monitor 1.5, Roche). Gonococcal infection among participants who reported urethral or rectal symptoms was defined as the detection of gram-negative, intracellular diplococci consistent with Neisseria gonorrhoeae in urethral or rectal secretions [7]. Prevalent syphilis infection was diagnosed using a positive rapid plasma reagin (RPR, tested annually) titre confirmed by Treponema pallidum hemagglutination assay (TPHA). Incident syphilis was defined as a fourfold increase in RPR titre confirmed by TPHA [7].

\section{Measures}

Four risk behaviors that had been previously associated with HIV-1 acquisition among this population were the primary predictors [7]. The first—-sexual activity and condom use in the past week-was categorized in three groups: "no activity," when no sex was reported in the past week; "all protected," when the number of sexual acts (either vaginal or anal intercourse) for which a condom was used was equal to the number of sexual acts reported; and "any unprotected," when the number of sexual acts for which a condom was used was lower than the number of acts reported. For the second variable, men were asked at each visit to report whether they had been sexually active with men, women, or both men and women in the past 3 months or since their last visit. This variable was categorized as "men only," "both men and women," and "women only." The third and fourth variables-having RAI and having group sex (defined as sex 
with more than one person at the same time)—were assessed for the past 3 months, or since the last visit, and categorized as either yes or no.

Other variables evaluated as potential predictors of HIV-1 acquisition included sociodemographic data collected at enrollment (age, education level, religion, marital and employment status) and time-updated data collected at each study visit (specific sexual behaviors, transactional sex [ 2 variables, defined as either paying for or being paid for sex with cash, living expenses, or goods], alcohol use, having sex after alcohol use, intravenous drug use, sharing needles among intravenous drug users, use of post-exposure prophylaxis, medical injections, urethral discharge or dysuria, rectal discharge, circumcision status, and self-reported genital sores).

\section{Data Analysis and Statistical Methods}

Descriptive statistics were used to summarize baseline sociodemographic and behavioral characteristics of MSM enrolled in the study. Data for each participant were censored at the end of 2016, the last visit for those who became lost to follow-up, or, for those who acquired HIV-1 infection during follow-up, the last seronegative and HIV-1 RNA-negative visit. Total observation time was obtained by adding up separate observation times for all participants in the study, and expressed in terms of PY. HIV-1 incidence rates were calculated as the number of HIV-1 incidence cases divided by PY of follow-up, and expressed as incidence per 100 PY. All potential predictors were assessed for association with incidence of HIV-1 infection using Poisson models with robust standard errors, to obtain population-averaged incidencerate ratios that accounted for correlation due to repeated measurements of the same subject over time. Variables significant at $P \leq 0.1$ in bivariable analysis were included in an initial multivariable model of potential predictors for HIV-1 acquisition. Variables with $P>0.1$ in the initial multivariable model were then dropped to produce a final multivariable model.

\section{Development of Empiric Risk Score}

Predictors in the final multivariable model were used to develop an empiric, model-based risk score, after excluding religion and insertive anal intercourse (IAI) (both of which were negatively associated with HIV-1 acquisition and would have led to negative risk scores for some men). A risk score was assigned to each statistically significant predictor for HIV-1 acquisition, based on its coefficient in the risk score model, and rounded to the nearest integer. Risk scores were then summed to generate predictor scores at a given visit for each participant. Crude HIV-1 incidence was calculated by different risk score cutoff values (e.g., $\geq 0, \geq 1$ ) to determine which risk score cutoff value correlated most closely with the HIV-1 incidence of $\geq 3$ per 100 PY recommended for PrEP initiation. To calculate the proportion of MSM who should be targeted for PrEP based on this risk score cutoff, we assessed risk over the previous 3 months at the last follow-up visit, as this would best reflect current risk behavior. We assessed whether higher risk scores correlated with higher HIV-1 incidence using a nonparametric test for trend. We evaluated risk score performance by assessing sensitivity, specificity, and area under the receiver operating characteristic curve (AUC). $P$ values were 2 -sided, and significance was set at $P \leq 0.05$. Data were cleaned, recoded, and analyzed using Stata 15.0 (StataCorp LLC, College Station, Texas, USA).

\section{Ethical Considerations}

The Kenya Medical Research Institute Ethics Review Committee approved this study. All participants provided written informed consent.

\section{Results}

\section{Study Population and Follow-up Duration}

A total of 753 HIV-1 negative MSM had at least 1 follow-up visit and contributed 1378.5 PY (median 14.3, interquartile range 5.6-31.8 months). Table 1 presents the sociodemographic characteristics of the study population. At enrollment, the majority were 18-24 years of age and had never been married. At this visit, $314(41.7 \%)$ reported having only male sex partners, $451(59.9 \%)$ reported having any unprotected sex in the past week, $523(69.5 \%)$ reported having RAI, 523 (69.5\%) reported having IAI, and 121 (16.1\%) reported having group sex in the past 3 months. Thirty percent had paid for sex in the past 3 months, and nearly $70 \%$ had received payment for sex. Almost two-thirds had used alcoholic beverages in the past month, and more than half had had sex after using alcohol. The vast majority (94.2\%) were circumcised, $92(12.2 \%)$ reported urethral discharge or dysuria, 37 (4.9\%) reported rectal discharge, and $11(1.5 \%)$ had a prevalent gonorrhea infection diagnosed at enrollment.

\section{HIV-1 Incidence Estimates and Risk Factors for HIV-1 Acquisition}

Ninety-seven MSM acquired HIV-1 during follow-up, for an estimated HIV-1 incidence rate of 7.0 (95\% CI 5.8-8.6) per 100 PY. In bivariable analysis, HIV-1 acquisition was associated with age (18-34 years), never being married, not being Muslim, and with reporting at a study visit having only male sex partners, having any unprotected sex, having RAI, having 
Table 1 Baseline

sociodemographic and behavioral characteristics of 753 HIV-1 negative MSM in Kilifi,

Kenya, 2005-2016

\begin{tabular}{|c|c|c|}
\hline Characteristics & Overall $(\mathrm{n}=753)$ & $\mathrm{n}(\%)$ \\
\hline \multicolumn{3}{|l|}{ Age group (years) } \\
\hline $18-24$ & 408 & $(54.2)$ \\
\hline $25-34$ & 288 & $(38.2)$ \\
\hline $35+$ & 57 & $(7.6)$ \\
\hline \multicolumn{3}{|l|}{ Education } \\
\hline Primary/none & 361 & $(47.9)$ \\
\hline Secondary & 312 & $(41.4)$ \\
\hline Tertiary/higher & 80 & $(10.6)$ \\
\hline \multicolumn{3}{|l|}{ Marital status } \\
\hline Never married & 641 & $(85.1)$ \\
\hline Ever married & 112 & $(14.9)$ \\
\hline \multicolumn{3}{|l|}{ Religion } \\
\hline Christian & 366 & $(48.6)$ \\
\hline Muslim & 216 & $(28.7)$ \\
\hline Other/none & 171 & $(22.7)$ \\
\hline \multicolumn{3}{|l|}{ Employment } \\
\hline None & 267 & $(35.5)$ \\
\hline Self & 377 & $(50.1)$ \\
\hline Formal & 109 & $(14.5)$ \\
\hline \multicolumn{3}{|l|}{ Sex of partner in past 3 months $^{\mathrm{a}}$} \\
\hline Men only & 314 & $(41.7)$ \\
\hline Both men and women & 436 & $(57.9)$ \\
\hline Women only & 3 & $(0.4)$ \\
\hline \multicolumn{3}{|l|}{ Sexual exposure and protection with condoms in past week } \\
\hline No activity & 133 & $(17.7)$ \\
\hline All protected & 169 & $(22.4)$ \\
\hline Any unprotected & 451 & $(59.9)$ \\
\hline Receptive anal intercourse (RAI) in past 3 months ${ }^{\mathrm{b}}$ & 523 & $(69.5)$ \\
\hline Insertive anal intercourse (IAI) in past 3 months $^{\mathrm{b}}$ & 523 & $(69.5)$ \\
\hline Paid for sex with cash, living expenses, or goods in past 3 months & 226 & $(30.0)$ \\
\hline $\begin{array}{l}\text { Received payment for sex with cash, living expenses, or goods in past } \\
3 \text { months }\end{array}$ & 523 & $(69.5)$ \\
\hline Group sex in past 3 months & 121 & $(16.1)$ \\
\hline Alcohol use in past month & 482 & $(64.0)$ \\
\hline Sex after alcohol use in past month & 405 & $(53.8)$ \\
\hline Been raped in past 3 months & 15 & $(2.0)$ \\
\hline Intravenous drug use in past 3 months & 12 & $(1.6)$ \\
\hline Sharing needles among intravenous drug users & 3 & $(0.4)$ \\
\hline Use of post-exposure prophylaxis & 1 & $(0.1)$ \\
\hline Medical injection in past 3 months & 57 & $(7.6)$ \\
\hline Urethral discharge or dysuria & 92 & $(12.2)$ \\
\hline Rectal discharge & 37 & $(4.9)$ \\
\hline Lab-confirmed gonorrhea infection $^{c}$ & 11 & $(1.5)$ \\
\hline Genital sores in past 3 months & 54 & $(7.2)$ \\
\hline Prevalent syphilis & 8 & $(1.1)$ \\
\hline Circumcised $^{\mathrm{d}}$ & 709 & $(94.2)$ \\
\hline
\end{tabular}

${ }^{a}$ Three men reported sex with women only at enrollment, and sex with both men and women during followup

${ }^{\mathrm{b}}$ Forty-six percent reported both RAI and IAI

${ }^{\mathrm{c} B a s e d}$ on gram staining of secretions collected from 118 volunteers who had urethral discharge or dysuria or rectal discharge

${ }^{\mathrm{d}}$ Missing 1 value for circumcision 
IAI, having paid for sex, having been paid for sex, and having group sex (Table 2). In the final multivariable model, HIV-1 acquisition was higher among MSM who were young (18-24 years) (adjusted incidence rate ratio [aIRR] 4.2, 95\% CI 1.2-14.2) and among those who reported having only male sex partners (aIRR 2.0, 95\% CI 1.1-3.4), any unprotected sex (aIRR 2.9, 95\% CI, 1.7-4.8), RAI (aIRR 4.6, 95\% CI 2.3-9.1), and group sex (aIRR 2.3, 95\% CI 1.4-3.9). Incidence was lower among men who were Muslim (aIRR 0.3, 95\% CI 0.2-0.6) and among men who reported having IAI (aIRR 0.6, 95\% CI 0.4-1.0) (Table 2).

\section{Empiric Risk Score Model}

Table 3 presents the risk score model, which excluded religion and IAI for reasons explained above. A risk score of 1, based on coefficients, was applied for each of these independent predictors of HIV-1 infection: younger age (18-24 years), having only male sex partners, having RAI, having any unprotected sex, and having group sex (Table 3). While the maximum score was 5 , only a very small proportion $(\sim 1.0 \%)$ of MSM visits were in this category (data not shown). We combined visits that had risk scores of 4 and 5 , to avoid wide confidence intervals due to sparse data. A risk score cutoff of $\geq 1$ corresponded to an HIV-1 incidence of $\geq 2.2$ (95\% CI 1.2-4.1) per 100 PY (Table 4 ), and had a sensitivity of $\leq 97.9 \%$ and a specificity of $\leq 16.9 \%$ for detecting the visit at which a participant had acquired HIV-1. If this cutoff is applied to predictors assessed at the last visit of men participating in the cohort, approximately $81.3 \%$ of MSM would be identified as high-risk individuals who should be targeted for PrEP initiation. The AUC for predictive ability of the risk score was 0.76 (95\% CI 0.71-0.80), indicating fair to good performance in identifying men who eventually seroconverted. A unit increase in risk score strongly correlated with an increase in observed HIV-1 incidence $(P<0.001$, test for trend) (Fig. 1).

\section{Assessing PrEP Eligibility}

Among the 753 MSM assessed at their last cohort visit for PrEP eligibility, 618 (82.1\%) would qualify for PrEP per criteria in the national guidelines. Of the 135 who would not qualify for PrEP, 86 (63.1\%) MSM, including 10 who had acquired HIV-1 infection, would qualify for PrEP based on their risk score in this study. Of these 86 PrEP-eligible MSM, the majority $(72.1 \%)$ reported having only male sex partners or having RAI.

\section{Discussion}

We developed an empiric risk score for PrEP targeting of MSM based on 5 predictors of HIV-1 acquisition derived from our MSM cohort. Adding 1 point for each of the predictors present-having only male sex partners, RAI, any unprotected sex, group sex, and young age (18-24 years) - led to a score that strongly correlated with increased HIV-1 incidence and had a fair to good predictive ability. By applying this risk score to MSM at their last cohort visit, we identified more than $80 \%$ of MSM as being at high risk for HIV-1 acquisition, with scores corresponding to an HIV-1 incidence as high as 4.1 per 100 PY (upper limit of our confidence interval).

Importantly, this tool identified 86 men $(11.4 \%$ of the cohort) as being at high risk for HIV-1 acquisition yet they did not meet PrEP eligibility criteria per Kenyan guidelines. In Kenya, PrEP guidelines do not specifically target individuals who report having RAI, only male sex partners, or group sex-predictors that were strongly associated with HIV-1 acquisition among MSM in coastal Kenya [7]. While many MSM do qualify for PrEP based on other risk behaviors, the application of our risk score, which includes young age and sexual behaviors that increase vulnerability among MSM, will assist providers in identifying those who are likely to benefit from PrEP initiation; therefore, our model should be beneficial to HIV-1 prevention programming in Kenya.

In Kenya, homosexuality is illegal and punishable by law [25]. MSM are stigmatized and face challenges in accessing health care services [26]. Although efforts have been made to train health care workers on the needs of MSM, trained providers themselves have reported feeling stigmatized by community members and other health care staff [27]. Despite these challenges, more than 1200 health care providers have taken an MSM sensitivity training that is freely available online (www.marps-africa.org) [28], and various programs engage MSM for PrEP programming in Kisumu, Nairobi, and coastal Kenya. Unfortunately, stigma surrounding male-male sex and RAI may have led to the omission of these factors as risk criteria in the Kenyan guidelines.

The empiric risk scoring tool had fair to good performance (AUC 0.76) in detecting HIV-1 among MSM in our cohort. Although the scoring tool has not been validated elsewhere, our aim was to develop a tool that could guide health care providers in both targeting MSM at highest risk for HIV-1 acquisition and discussing the risks that should prompt PrEP initiation. This scoring tool could potentially be used by clinicians and counselors to reevaluate PrEP eligibility among MSM already on PrEP and guide a discussion on continuation or discontinuation. Outside the clinical 
Table 2 Risk factors for HIV-1 acquisition among 753 MSM in Kilifi, Kenya, 2005-2016

\begin{tabular}{|c|c|c|c|c|c|c|c|}
\hline \multirow[t]{2}{*}{ Characteristics $^{\mathrm{a}}$} & \multirow[t]{2}{*}{ Incidence/100 PY (95\% CI) } & \multicolumn{2}{|c|}{ Bivariable analysis } & \multicolumn{2}{|c|}{ Initial multivariable model } & \multicolumn{2}{|c|}{ Final multivariable model } \\
\hline & & IRR (95\% CI) & $P$ value & $\operatorname{aIRR}(95 \% \mathrm{CI})^{\mathrm{b}}$ & $P$ value & $\operatorname{aIRR}(95 \% \mathrm{CI})^{\mathrm{c}}$ & $P$ value \\
\hline All men & $7.0(5.8-8.6)$ & & & & & & \\
\hline \multicolumn{8}{|l|}{ Age categories (years) } \\
\hline $18-24$ & $10.6(8.1-13.8)$ & $6.4(2.0-21.1)$ & 0.002 & $4.2(1.1-15.9)$ & 0.034 & $4.2(1.2-14.2)$ & 0.023 \\
\hline $25-34$ & $5.8(4.3-7.9)$ & $3.5(1.1-11.7)$ & 0.038 & $2.6(0.7-9.8)$ & 0.157 & $2.6(0.8-8.9)$ & 0.125 \\
\hline $35+$ & $1.6(0.5-5.1)$ & Reference & & Reference & & Reference & \\
\hline \multicolumn{8}{|l|}{ Education } \\
\hline Primary/none & $6.2(4.6-8.3)$ & Reference & & Reference & & & \\
\hline Secondary & $7.3(5.3-10.0)$ & $1.2(0.7-1.9)$ & 0.471 & $0.8(0.5-1.4)$ & 0.494 & - & - \\
\hline Tertiary/higher & $11.3(6.6-19.5)$ & $1.8(0.9-3.6)$ & 0.075 & $1.3(0.6-2.5)$ & 0.521 & - & - \\
\hline \multicolumn{8}{|l|}{ Marital status } \\
\hline Never married & $7.8(6.4-9.6)$ & Reference & & Reference & & & \\
\hline Ever married & $3.1(1.5-6.4)$ & $0.4(0.2-0.9)$ & 0.019 & $1.2(0.5-3.0)$ & 0.715 & - & - \\
\hline \multicolumn{8}{|l|}{ Religion } \\
\hline Christian & $8.7(6.7-11.3)$ & Reference & & Reference & & Reference & \\
\hline Muslim & $4.2(2.7-6.5)$ & $0.5(0.3-0.8)$ & 0.009 & $0.4(0.2-0.7)$ & 0.001 & $0.3(0.2-0.6)$ & 0.001 \\
\hline None/other & $8.0(5.2-12.2)$ & $0.9(0.5-1.5)$ & 0.731 & $0.8(0.5-1.4)$ & 0.403 & $0.8(0.5-1.4)$ & 0.413 \\
\hline \multicolumn{8}{|l|}{ Employment } \\
\hline None & $6.7(4.9-9.2)$ & Reference & & & & & \\
\hline Self & $6.5(4.8-8.9)$ & $1.0(0.6-1.6)$ & 0.922 & - & - & & \\
\hline Formal & $9.5(6.1-14.9)$ & $1.4(0.8-2.6)$ & 0.259 & - & - & & \\
\hline \multicolumn{8}{|c|}{ Sex of partner in past 3 months } \\
\hline Both men and women & $3.4(2.4-4.9)$ & Reference & & Reference & & Reference & \\
\hline Men only & $14.6(11.4-18.6)$ & $4.3(2.7-6.6)$ & $<0.001$ & $1.9(1.1-3.3)$ & 0.026 & $2.0(1.1-3.4)$ & 0.016 \\
\hline \multicolumn{8}{|c|}{ Sexual exposure and protection with condoms in past week } \\
\hline No activity & $3.5(2.1-5.7)$ & Reference & & Reference & & Reference & \\
\hline All protected & $5.9(4.0-8.7)$ & $1.7(0.9-3.2)$ & 0.099 & $1.3(0.7-2.3)$ & 0.462 & $1.2(0.7-2.2)$ & 0.573 \\
\hline Any unprotected & $11.5(8.8-14.9)$ & $3.3(1.9-5.8)$ & $<0.001$ & $3.0(1.8-5.1)$ & $<0.001$ & $2.9(1.7-4.8)$ & $<0.001$ \\
\hline \multicolumn{8}{|c|}{ Receptive anal intercourse (RAI) in past 3 months } \\
\hline No & $1.8(1.0-3.0)$ & Reference & & Reference & & Reference & \\
\hline Yes & $13.2(10.7-16.4)$ & $7.5(4.2-13.6)$ & $<0.001$ & $4.4(2.2-9.0)$ & $<0.001$ & $4.6(2.3-9.1)$ & $<0.001$ \\
\hline \multicolumn{8}{|c|}{ Insertive anal intercourse (IAI) in past 3 months } \\
\hline No & $9.0(6.9-11.7)$ & Reference & & Reference & & Reference & \\
\hline Yes & $5.5(4.1-7.5)$ & $0.6(0.4-0.9)$ & 0.021 & $0.6(0.4-1.0)$ & 0.057 & $0.6(0.4-1.0)$ & 0.044 \\
\hline \multicolumn{8}{|c|}{ Paid for sex with cash, living expenses, or goods in past 3 months } \\
\hline No & $8.1(6.6-10.0)$ & Reference & & Reference & & & \\
\hline Yes & $3.6(2.1-6.4)$ & $0.5(0.2-0.8)$ & 0.011 & $0.7(0.4-1.3)$ & 0.273 & - & - \\
\hline \multicolumn{8}{|c|}{ Received payment for sex with cash, living expenses, or goods in past 3 months } \\
\hline No & $4.8(3.4-6.8)$ & Reference & & Reference & & & \\
\hline Yes & $9.1(7.2-11.7)$ & $1.9(1.2-2.9)$ & 0.004 & $0.9(0.6-1.5)$ & 0.821 & - & - \\
\hline \multicolumn{8}{|c|}{ Group sex in past 3 months } \\
\hline No & $6.0(4.8-7.5)$ & Reference & & Reference & & Reference & \\
\hline Yes & $19.6(12.8-30.1)$ & $3.3(2.0-5.3)$ & $<0.001$ & $2.4(1.4-4.0)$ & 0.001 & $2.3(1.4-3.9)$ & 0.001 \\
\hline \multicolumn{8}{|c|}{ Alcoholic use in past month } \\
\hline No & $7.3(5.4-9.9)$ & Reference & & & & & \\
\hline Yes & $6.8(5.3-8.9)$ & $0.9(0.6-1.4)$ & 0.755 & - & - & & \\
\hline \multicolumn{8}{|c|}{ Sex after alcohol use in past month } \\
\hline No & $7.4(5.7-9.6)$ & Reference & & & & & \\
\hline Yes & $6.6(4.8-9.0)$ & $0.9(0.6-1.3)$ & 0.579 & - & - & - & \\
\hline
\end{tabular}


Table 2 (continued)

\begin{tabular}{|c|c|c|c|c|c|c|c|}
\hline \multirow[t]{2}{*}{ Characteristics $^{\mathrm{a}}$} & \multirow[t]{2}{*}{ Incidence/100 PY (95\% CI) } & \multicolumn{2}{|c|}{ Bivariable analysis } & \multicolumn{2}{|c|}{ Initial multivariable model } & \multicolumn{2}{|c|}{ Final multivariable model } \\
\hline & & IRR $(95 \% \mathrm{CI})$ & $P$ value & $\operatorname{aIRR}(95 \% \mathrm{CI})^{\mathrm{b}}$ & $P$ value & $\operatorname{aIRR}(95 \% \mathrm{CI})^{\mathrm{c}}$ & $P$ value \\
\hline \multicolumn{8}{|c|}{ Raped in past 3 months } \\
\hline No & $7.0(5.7-8.6)$ & Reference & & & & & \\
\hline Yes & $10.8(1.5-76.3)$ & $1.5(0.2-11.1)$ & 0.676 & - & - & & \\
\hline \multicolumn{8}{|c|}{ Intravenous drug use in past 3 months } \\
\hline No & $7.0(5.7-8.5)$ & Reference & & & & & \\
\hline Yes & $16.3(2.3-115.4)$ & $2.3(0.3-19.1)$ & 0.433 & - & - & & \\
\hline \multicolumn{8}{|c|}{ Had medical injections in past 3 months } \\
\hline No & $6.9(5.7-8.5)$ & Reference & & & & & \\
\hline Yes & $13.6(5.1-36.1)$ & $2.0(0.7-5.4)$ & 0.197 & - & - & & \\
\hline \multicolumn{8}{|c|}{ Urethral discharge or dysuria } \\
\hline No & $6.7(5.4-8.3)$ & Reference & & & & & \\
\hline Yes & $12.0(6.5-22.3)$ & $1.8(0.9-3.6)$ & 0.101 & - & - & - & - \\
\hline \multicolumn{8}{|l|}{ Rectal discharge } \\
\hline No & $6.9(5.6-8.5)$ & Reference & & & & & \\
\hline Yes & $15.8(5.1-49.0)$ & $2.3(0.7-7.5)$ & 0.179 & - & - & - & - \\
\hline \multicolumn{8}{|c|}{ Lab-confirmed gonorrhea infection } \\
\hline No & $6.9(5.7-8.5)$ & Reference & & Reference & & & \\
\hline Yes & $26.2(6.5-104.7)$ & $3.8(0.8-16.7)$ & 0.082 & $3.4(0.7-17.1)$ & 0.141 & - & - \\
\hline \multicolumn{8}{|c|}{ Genital sores in past 3 months } \\
\hline No & $7.0(5.7-8.5)$ & Reference & & Reference & & & \\
\hline Yes & $17.0(6.4-45.2)$ & $2.4(0.9-6.6)$ & 0.084 & $1.8(0.6-5.0)$ & 0.269 & - & - \\
\hline \multicolumn{8}{|c|}{ Circumcision status } \\
\hline No & $9.7(4.3-21.5)$ & $1.4(0.6-3.4)$ & 0.460 & - & - & & \\
\hline Yes & $6.9(5.6-8.5)$ & Reference & & & & & \\
\hline
\end{tabular}

$C I$ confidence interval, IRR incidence rate ratio, aIRR adjusted incidence rate ratio

a"Sharing needles among intravenous drug users," "use of post-exposure prophylaxis," and "syphilis infection" excluded, as no HIV-1 acquisitions occurred in the exposed categories

${ }^{\mathrm{b}}$ Only factors significant at $P \leq 0.1$ in the bivariable analysis were included in the initial multivariable model

${ }^{c}$ Only factors significant at $P \leq 0.1$ in the initial multivariable model were retained in the final multivariable model

setting, this risk scoring tool may be used by public health professionals offering HIV-1 and STI services for MSM or scaling up PrEP among MSM and other key populations.

Our study confirmed that having RAI, any unprotected sex, and group sex remained independent predictors for HIV-1 acquisition among our cohort, similar to the period of 2005-2011 [7]. We found a twofold higher incidence of HIV-1 acquisition among men who reported having only male sex partners, compared with men who reported both male and female partners, which was consistent with our previous reports [10]. MSM aged 18-24 years had a fourfold higher incidence of HIV-1 acquisition, compared with men older than 34 years. Elsewhere, in a Bangkok cohort, young MSM (aged $<21$ years) had an increased risk for HIV-1 acquisition $[29,30]$.

Men who reported having IAI were less likely to acquire HIV-1 infection than men who did not report IAI (5.5 vs 9.0 per $100 \mathrm{PY}$ ) in our study (Table 2). Although we did not specifically assess whether condoms were used for IAI, with whom IAI was practiced, or the frequency of IAI versus RAI, a protective association for IAI remained after adjusting for other risk factors for HIV-1 acquisition among our cohort. This finding is consistent with a lower per-act risk for HIV-1 transmission via IAI, compared with the risk via RAI [31], and may also result from a protective effect of circumcision during IAI [32].

We found that MSM who were Muslim had a lower HIV-1 incidence than MSM who were Christian (4.2 vs 8.7 per 100 PY, Table 2), which has not been reported previously [7]. Why MSM who are Muslim have lower HIV-1 acquisition risks is not clear. In our risk score model, we did not assign a negative risk score for MSM who reported having IAI or for MSM who were Muslim, as both groups already had substantial HIV-1 acquisition risks.

As national programs target key populations for PrEP initiation in Kenya, our risk score model may guide health care 
Table 3 Risk factors and corresponding risk scores for HIV-1 acquisition among 753 MSM in Kilifi, Kenya, 2005-2016

\begin{tabular}{|c|c|c|c|c|}
\hline Characteristics & $\begin{array}{l}\text { Risk score model IRR } \\
(95 \% \mathrm{CI})\end{array}$ & $P$ value & $\begin{array}{l}\text { Risk score model } \\
\text { coefficient }\end{array}$ & Risk score $^{\mathrm{a}}$ \\
\hline \multicolumn{5}{|l|}{ Age categories (years) } \\
\hline $18-24$ & $4.0(1.2-13.6)$ & 0.024 & 1.40 & 1 \\
\hline $25-34$ & $2.7(0.8-9.1)$ & 0.113 & 0.99 & - \\
\hline $35+$ & Reference & & Reference & \\
\hline \multicolumn{5}{|c|}{ Sex of partner in past 3 months } \\
\hline Both men and women & Reference & & Reference & \\
\hline Men only & $2.2(1.3-3.7)$ & 0.002 & 0.80 & 1 \\
\hline \multicolumn{5}{|c|}{ Sexual exposure and protection with condoms in past week } \\
\hline No activity & Reference & & Reference & \\
\hline All protected & $1.2(0.7-2.2)$ & 0.566 & 0.18 & - \\
\hline Any unprotected & $2.6(1.5-4.4)$ & $<0.001$ & 0.95 & 1 \\
\hline \multicolumn{5}{|c|}{ Receptive anal intercourse (RAI) in past 3 months } \\
\hline No & Reference & & Reference & \\
\hline Yes & $4.3(2.2-8.5)$ & $<0.001$ & 1.45 & 1 \\
\hline \multicolumn{5}{|c|}{ Group sex in past 3 months } \\
\hline No & Reference & & Reference & \\
\hline Yes & $1.9(1.1-3.3)$ & 0.014 & 0.67 & 1 \\
\hline
\end{tabular}

$C I$ confidence interval, IRR incidence rate ratio

${ }^{a}$ Coefficient from the risk score model rounded to the nearest integer

Table 4 HIV-1 incidence $(95 \% \mathrm{CI})$, sensitivity, specificity and proportion of men with a risk score at or above each cutoff point, among 753 MSM in Kilifi, Kenya, 2005-2016

\begin{tabular}{|c|c|c|c|c|c|c|}
\hline Risk score & $\begin{array}{l}\text { Cases/follow-up } \\
\text { time (PY) }\end{array}$ & HIV-1 incidence (95\% CI) & Sensitivity (\%) & Specificity (\%) & $\begin{array}{l}\text { Total no. of } \\
\text { MSM }^{\mathrm{a}}\end{array}$ & $\begin{array}{l}\text { Proportion at or } \\
\text { above cutoff point } \\
(\%)\end{array}$ \\
\hline 0 & $2 / 255.4$ & $0.8(0.2-3.1)$ & 100.0 & 0.0 & 141 & 100.0 \\
\hline 1 & $10 / 454.5$ & $2.2(1.2-4.1)$ & 97.9 & 16.9 & 231 & 81.3 \\
\hline 2 & $24 / 372.3$ & $6.4(4.3-9.6)$ & 87.6 & 48.3 & 198 & 50.6 \\
\hline 3 & $30 / 208.1$ & $14.4(10.1-20.6)$ & 62.9 & 76.0 & 124 & 24.3 \\
\hline$\geq 4$ & $31 / 88.2$ & $35.2(24.7-50.0)$ & 32.0 & 92.8 & 59 & 7.8 \\
\hline
\end{tabular}

$C I$ confidence interval, $P Y$ person-years

${ }^{a}$ Assessed at the last follow-up visit

providers in discussing specific behaviors that put MSM at increased risk for HIV-1 acquisition and are not included in the current NASCOP criteria for PrEP initiation. MSM with a risk score of $\geq 1$ should be made aware of their elevated risk for HIV-1 acquisition and encouraged to consider PrEP as an additional risk reduction strategy. Additionally, they should be evaluated for symptoms of acute HIV-1 infection before PrEP initiation [33, 34], in accordance with Kenyan national guidelines [3]. MSM who have an empiric risk score of $<1$ may be willing to take PrEP and may qualify for PrEP according to Kenyan guidelines for other factors (e.g., an ongoing relationship with an HIV-1-positive partner, transactional sex, or injection drug use with a needle-sharing partner) [3]. Our risk score model is intended as a supplement to the Kenya guidelines, not as a replacement for them.
Our study had several limitations. First, we did not collect data on partner characteristics, duration of partnerships, partner's HIV-1 status, or condom use by the participant or partner specifically during anal sex. Second, self-reported sexual behavior is subject to recall and social desirability biases, and may have led to underreporting of risky sexual behaviors during risk assessment. Third, our STI assessment was restricted to symptomatic patients (having urethral discharge or dysuria or having rectal discharge) only, so we were unable to detect asymptomatic infections. Fourth, the time-updated PrEP eligibility score reflects risks assessed over the previous 3 months. As risk is not static, PrEP eligibility should be assessed and periodically updated over time. Fifth, while we have identified factors associated with risk among this cohort, their importance and relative 


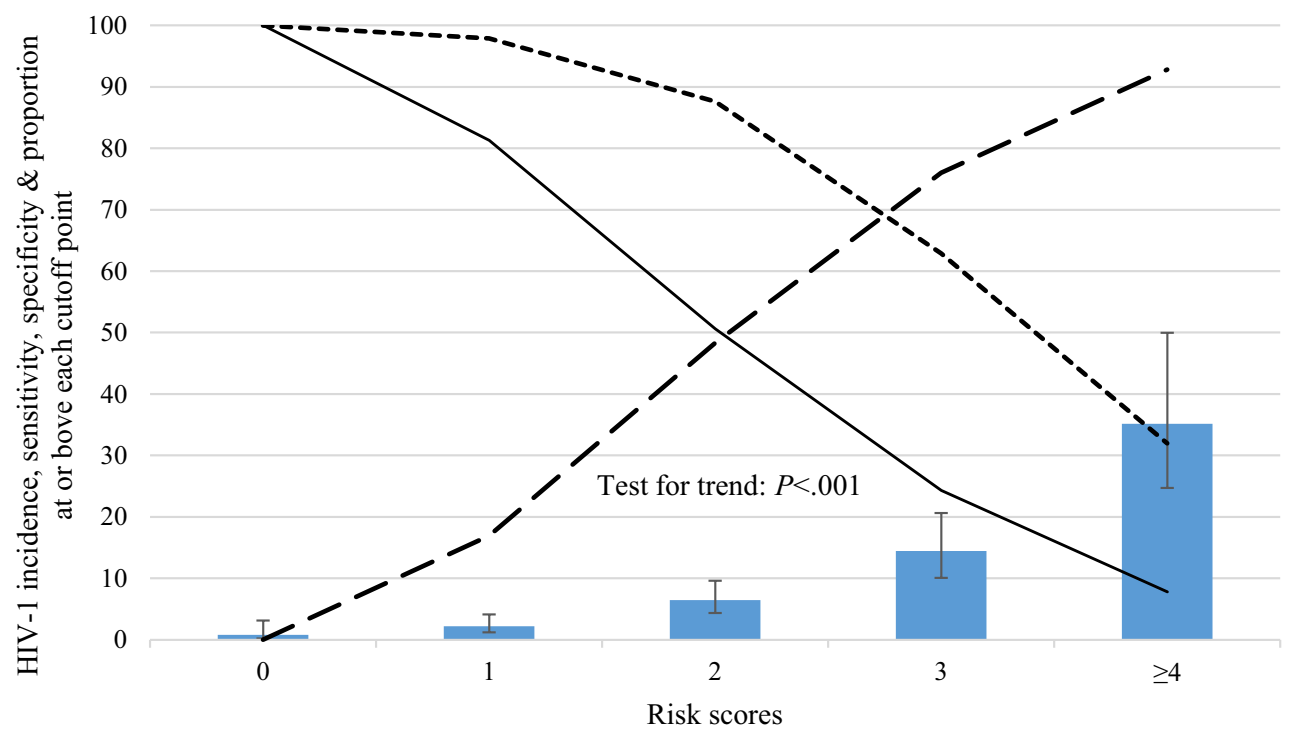

Inc - - - Sensitivity - Specificity - Proportion at or above cutoff point

Fig. 1 HIV-1 incidence (95\% CI), sensitivity, specificity and proportion of men with a risk score at or above each cutoff point, among 753 MSM in Kilifi, Kenya, 2005-2016

contribution to HIV-1 acquisition among different gay, bisexual, and other MSM populations in the future should be validated and periodically reassessed.

\section{Conclusion}

A simple risk score consisting of 5 characteristics can be used to identify MSM at highest risk for HIV-1 infection, who then should be prioritized for PrEP. This risk score may help Kenyan health care providers in discussing HIV-1 acquisition risk among MSM and encouraging them to consider PrEP when risk criteria are met. All MSM should be evaluated for symptoms of acute HIV-1 infection prior to PrEP initiation. Further work is needed to validate this risk scoring tool among other MSM cohorts.

Acknowledgements We would like to thank the participants and research team for their contributions to the study. We wish to acknowledge previous support to Elizabeth Wahome from the University of California, San Francisco, through its International Traineeships in AIDS Prevention Studies (ITAPS), US NIMH, R25MH064712, and the Starr Foundation Scholarship Fund. This work was also supported in part through the Sub-Saharan African Network for TB/HIV Research Excellence (SANTHE), a DELTAS Africa Initiative (Grant \# DEL-15006). The DELTAS Africa Initiative is an independent funding scheme of the African Academy of Sciences (AAS) Alliance for Accelerating Excellence in Science in Africa (AESA) and is supported by the New Partnership for Africa's Development Planning and Coordinating Agency (NEPAD Agency) with funding from the Wellcome Trust (Grant \# 107752/Z/15/Z) and the UK government. The views expressed in this publication are those of the authors and not necessarily those of AAS, NEPAD Agency, Wellcome Trust, or the UK government.
Funding This work was supported by the International AIDS Vaccine Initiative (IAVI) and the Universtity of Washington Center for AIDS Research, a National Institutes of Health (NIH)-Funded Program [R01AI124968], which is supported by the following NIH institutes and centers (NIAID, NCI, NIMH, NIDA, NICHD, NHLBI, NCCAM). Susan M. Graham was supported by 1R01AI124968-01A1. The KEMRI Wellcome Trust Research Programme at the Centre for Geographical Medicine Research-Kilifi is supported by core funding from the Wellcome Trust (Grant \# 203077/Z/16/Z). This study was made possible by the generous support of the American people through the United States Agency for International Development (USAID). The contents are the responsibility of the study authors and do not necessarily reflect the views of USAID, NIH, the United States government, or the Wellcome Trust. This report was published with permission from the director of KEMRI.

\section{Compliance with Ethical Standards}

Conflict of interest The authors declare that their research was conducted in the absence of any commercial or financial relationship that could be construed as a potential conflict of interest. This paper has not been submitted elsewhere. Preliminary results were presented at the Conference on Retroviruses and Opportunistic Infections (CROI) 2017 (\#1601).

Ethical Approval All procedures performed in studies involving human participants were in accordance with the ethical standards of the institutional and/or national research committee and with the 1964 Helsinki declaration and its later amendments or comparable ethical standards.

Informed Consent Informed consent was obtained from all individual participants included in the study.

Open Access This article is distributed under the terms of the Creative Commons Attribution 4.0 International License (http://creativeco 
mmons.org/licenses/by/4.0/), which permits unrestricted use, distribution, and reproduction in any medium, provided you give appropriate credit to the original author(s) and the source, provide a link to the Creative Commons license, and indicate if changes were made.

\section{References}

1. Fonner VA, Dalglish SL, Kennedy CE, et al. Effectiveness and safety of oral HIV preexposure prophylaxis for all populations. AIDS. 2016;30:1973-83.

2. WHO. WHO expands recommendation on oral pre-exposure prophylaxis of HIV infection (PrEP). http://www.who.int/hiv/ pub/prep/policy-brief-prep-2015/en/. Accessed Jan 142017.

3. Ministry of Health, National AIDS \& STI Control Programme. Guidelines on use of antiretroviral drugs for treating and preventing HIV infection in Kenya 2016. www.prepwatch.org/wp-conte nt/uploads/2016/08/Guidelines-on-ARV-for-Treating-Preventing -HIV-Infections-in-Kenya.pdf. Accessed July 62017.

4. National AIDS \& STI control programme. http://www.nascop.or. ke/. Accessed July 62017.

5. NASCOP. Framework for the implementation of pre-exposure prophylaxis of HIV in Kenya. http://www.prepwatch.org/wp-conte nt/uploads/2017/05/Kenya_PrEP_Implementation_Framework-1. pdf. Accessed July 62017.

6. McKinnon LR, Gakii G, Juno JA, et al. High HIV risk in a cohort of male sex workers from Nairobi, Kenya. Sex Transm Infect. 2013;90(3):237-42.

7. Sanders EJ, Okuku HS, Smith AD, et al. High HIV-1 incidence, correlates of HIV-1 acquisition, and high viral loads following seroconversion among men who have sex with men in Coastal Kenya. AIDS. 2013;27:437.

8. Sanders EJ, Thiong'o AN, Okuku HS, et al. High prevalence of Chlamydia trachomatis and Neisseria gonorrhoeae infections among HIV-1 negative men who have sex with men in coastal Kenya. Sex Transm Infect. 2010;86:440-1.

9. Price MA, Rida W, Mwangome M, et al. Identifying at-risk populations in Kenya and South Africa: HIV incidence in cohorts of men who report sex with men, sex workers, and youth. J Acquir Immune Defic Syndr. 2012;59:185-93.

10. Sanders EJ, Graham SM, Okuku HS, et al. HIV-1 infection in high risk men who have sex with men in Mombasa, Kenya. AIDS. 2007;21:2513-20.

11. Tanser F, de Oliveira T, Maheu-Giroux M, Barnighausen T. Concentrated HIV subepidemics in generalized epidemic settings. Curr Opin HIV AIDS. 2014;9:115-25.

12. Cowan FM, Delany-Moretlwe S, Sanders EJ, et al. PrEP implementation research in Africa: what is new? J Int AIDS Soc. 2016. https://doi.org/10.7448/IAS.19.7.21101.

13. Buchbinder SP, Glidden DV, Liu AY, et al. HIV pre-exposure prophylaxis in men who have sex with men and transgender women: a secondary analysis of a phase 3 randomised controlled efficacy trial. Lancet Infect Dis. 2014;14:468-75.

14. Kahle EM, Hughes JP, Lingappa JR, et al. An empiric risk scoring tool for identifying high-risk heterosexual HIV-1 serodiscordant couples for targeted HIV-1 prevention. J Acquir Immune Defic Syndr. 1999;2013(62):339.

15. Irungu EM, Heffron $\mathrm{R}$, Mugo $\mathrm{N}$, et al. Use of a risk scoring tool to identify higher-risk HIV-1 serodiscordant couples for an antiretroviral-based HIV-1 prevention intervention. BMC Infect Dis. 2016;16:571.

16. Balkus JE, Brown E, Palanee T, et al. An empiric HIV risk scoring tool to predict HIV-1 acquisition in African women. J Acquir Immune Defic Syndr (1999). 2016;72(3):333-43.
17. Kagaayi J, Gray RH, Whalen C, et al. Indices to measure risk of HIV acquisition in Rakai, Uganda. PLoS ONE. 2014;9:e92015.

18. Pintye J, Drake AL, Kinuthia J, et al. A risk assessment tool for identifying pregnant and postpartum women who may benefit from pre-exposure prophylaxis (PrEP). Clin Infect Dis. 2016;64(6):751-8.

19. Hoenigl M, Weibel N, Mehta SR, et al. Development and validation of the San Diego Early Test Score to predict acute and early HIV infection risk in men who have sex with men. Clin Infect Dis. 2015;61:468-75.

20. Wilton J, Kain T, Fowler S, et al. Use of an HIV-risk screening tool to identify optimal candidates for PrEP scale-up among men who have sex with men in Toronto, Canada: disconnect between objective and subjective HIV risk. J Int AIDS Soc. 2016;19(1):20777.

21. Menza TW, Hughes JP, Celum CL, Golden MR. Prediction of HIV acquisition among men who have sex with men. Sex Transm Dis. 2009;36:547.

22. Smith DK, Pals SL, Herbst JH, Shinde S, Carey JW. Development of a clinical screening index predictive of incident HIV infection among men who have sex with men in the United States. J Acquir Immune Defic Syndr. 2012;60:421-7.

23. Beymer MR, Weiss RE, Sugar CA, et al. Are centers for disease control and prevention guidelines for preexposure prophylaxis specific enough? Formulation of a personalized HIV risk score for pre-exposure prophylaxis initiation. Sex Transm Dis. 2017;44:49-57.

24. Wahome E, Ngetsa C, Mwambi J, et al. Hepatitis B virus incidence and risk factors among human immunodeficiency virus-1 negative men who have sex with men in Kenya. Open Forum Infect Dis. 2017;4:OFW253.

25. Congress LLo. criminal laws on homosexuality in African Nations. https://www.loc.gov/law/help/criminal-laws-on-homos exuality/homosexuality-laws-in-african-nations.pdf. Accessed Nov 262017.

26. Smith AD, Tapsoba P, Peshu N, Sanders EJ, Jaffe HW. Men who have sex with men and HIV/AIDS in sub-Saharan Africa. Lancet. 2009;374:416-22.

27. van der Elst EM, Gichuru E, Omar A, et al. Experiences of Kenyan healthcare workers providing services to men who have sex with men: qualitative findings from a sensitivity training programme. J Int AIDS Soc. 2013;16(suppl 3):18741.

28. van der Elst EM, Gichuru E, Muraguri N, et al. Strengthening healthcare providers' skills to improve HIV services for MSM in Kenya. AIDS. 2015;29(suppl 3):S237-40.

29. van Griensven F, Holtz TH, Thienkrua W, et al. Temporal trends in HIV-1 incidence and risk behaviours in men who have sex with men in Bangkok, Thailand, 2006-13: an observational study. Lancet HIV. 2015;2:e64-70.

30. Thienkrua W, van Griensven F, Mock PA, et al. Young men who have sex with men at high risk for HIV, Bangkok MSM cohort study, Thailand 2006-2014. AIDS Behav. 2017. https://doi. org/10.1007/s10461-017-1963-7.

31. Patel P, Borkowf CB, Brooks JT, Lasry A, Lansky A, Mermin J. Estimating per-act HIV transmission risk: a systematic review. AIDS. 2014;28:1509-19.

32. Qian HZ, Ruan Y, Liu Y, et al. Lower HIV risk among circumcised men who have sex with men in China: interaction with anal sex role in a cross-sectional study. J Acquir Immune Defic Syndr. 2016;71:444-51.

33. Grant RM, Lama JR, Anderson PL, et al. Preexposure chemoprophylaxis for HIV prevention in men who have sex with men. $\mathrm{N}$ Engl J Med. 2010;363:2587-99.

34. Baeten JM, Haberer JE, Liu AY, Sista N. Preexposure prophylaxis for HIV prevention: where have we been and where are we going? J Acquir Immune Defic Syndr. 2013;63(suppl 2):S122-9. 